\title{
Editorial
}

\section{New Trends on Nonlocal and Functional Boundary Value Problems}

\author{
Gennaro Infante, ${ }^{1}$ To Fu Ma, ${ }^{2}$ and Feliz Manuel Minhós ${ }^{3}$ \\ ${ }^{1}$ Dipartimento di Matematica e Informatica, Università della Calabria, Arcavacata di Rende, 87036 Cosenza, Italy \\ ${ }^{2}$ Instituto de Ciências Matemáticas e de Computação, Universidade de São Paulo, 13566-560 São Carlos, SP, Brazil \\ ${ }^{3}$ Department of Mathematics, University of Évora, Rua Romão Ramalho 59, 7000-671 Évora, Portugal
}

Correspondence should be addressed to G. Infante; gennaro.infante@unical.it

Received 2 September 2013; Accepted 2 September 2013

Copyright (C) 2013 G. Infante et al. This is an open access article distributed under the Creative Commons Attribution License, which permits unrestricted use, distribution, and reproduction in any medium, provided the original work is properly cited.

In the last decades, boundary value problems with nonlocal and functional boundary conditions have become a rapidly growing area of research. The study of this type of problems not only has a theoretical interest that includes a huge variety of differential, integrodifferential, and abstract equations, but also is motivated by the fact that these problems can be used as a model for several phenomena in engineering, physics, and life sciences that standard boundary conditions cannot describe. In this framework, fall problems with feedback controls, such as the steady states of a thermostat, where a controller at one of its ends adds or removes heat depending upon the temperature registered in another point, or phenomena with functional dependence in the equation and/or in the boundary conditions, with delays or advances, maximum or minimum arguments, such as beams where the maximum (minimum) of the deflection is attained in some interior or endpoint of the beam. Topological and functional analysis tools, for example, degree theory, fixed point theorems, or variational principles, have played a key role in the developing of this subject.

This volume contains a variety of contributions within this area of research. The articles deal with second and higher order boundary value problems with nonlocal and functional conditions for ordinary, impulsive, partial, and fractional differential equations on bounded and unbounded domains. In the contributions, existence, uniqueness, and asymptotic behaviour of solutions are considered by using several methods as fixed point theorems, spectral analysis, and oscillation theory.

\section{Acknowledgments}

We would like to thank the authors for their contributions and the anonymous referees for their professional support.

Gennaro Infante

To Fu Ma

Feliz Manuel Minhós 


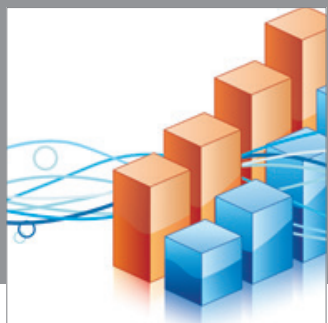

Advances in

Operations Research

mansans

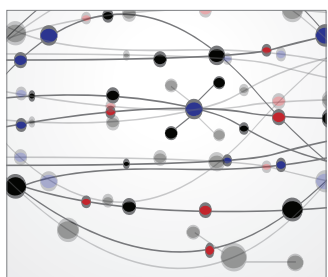

The Scientific World Journal
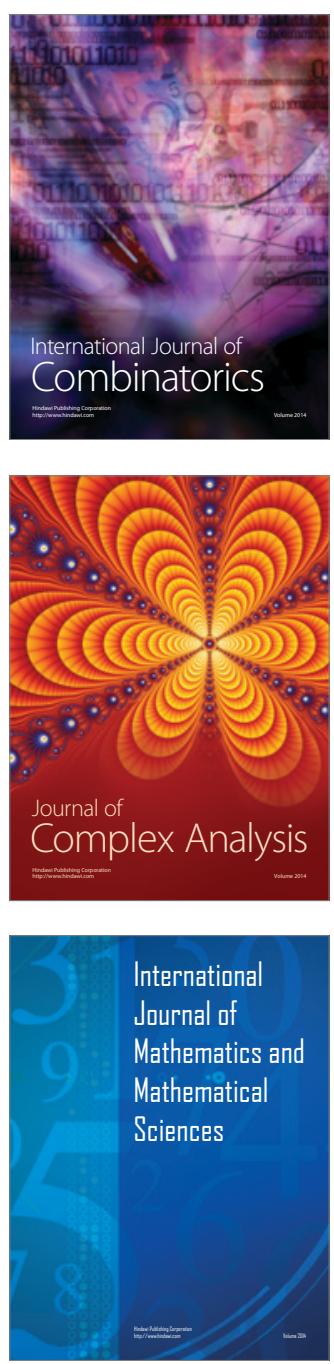
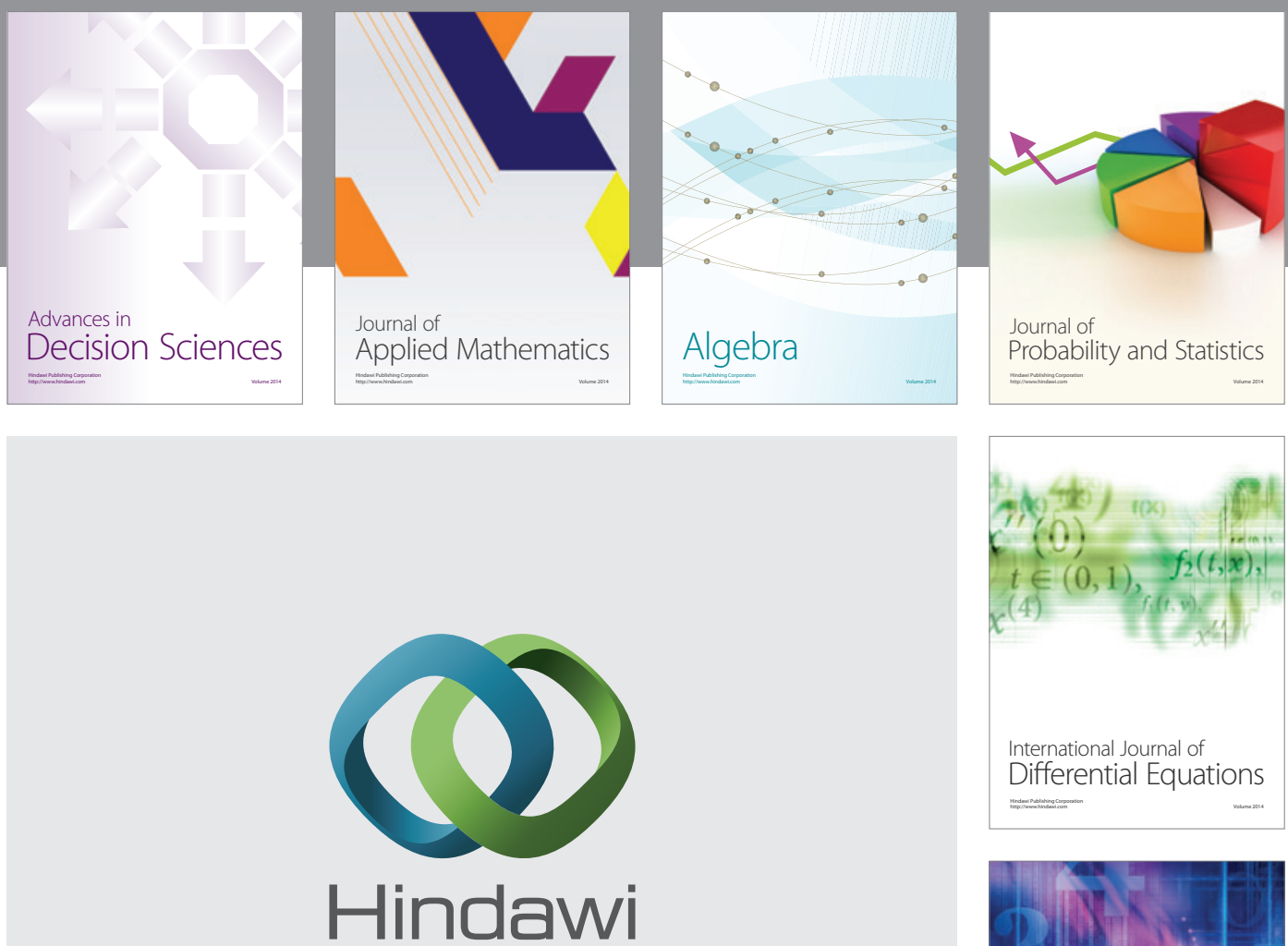

Submit your manuscripts at http://www.hindawi.com
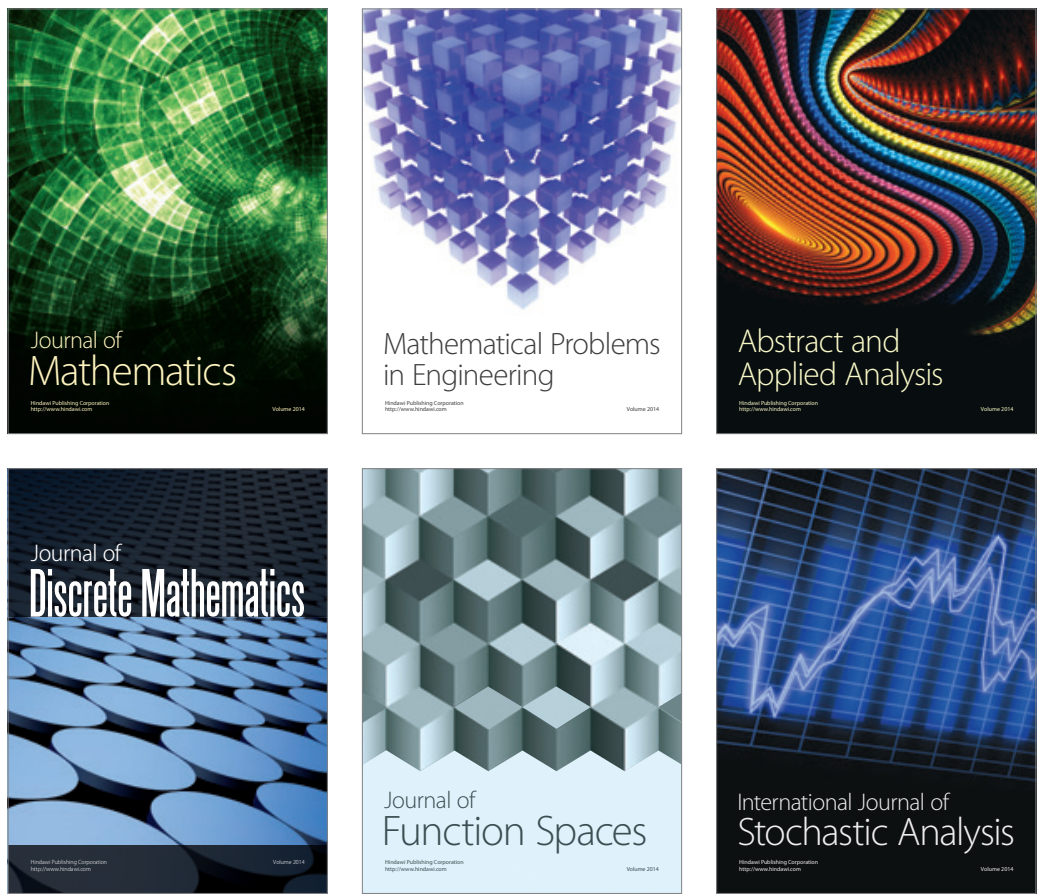

Journal of

Function Spaces

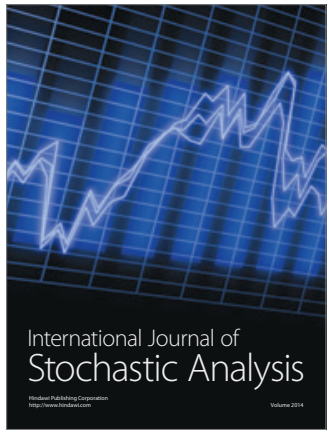

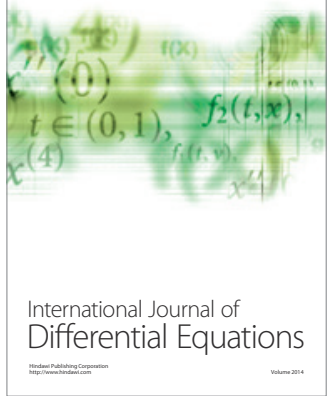
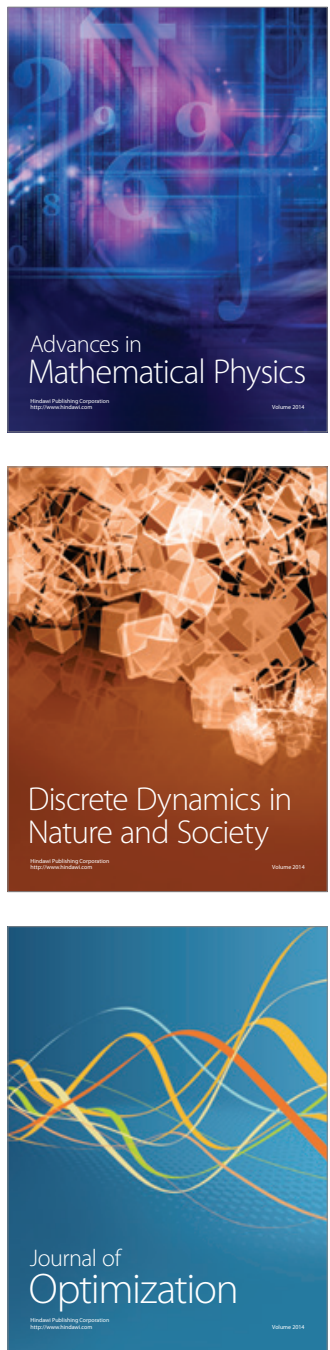